OPEN ACCESS

Edited by:

Mohammad Khursheed Alam, Al Jouf University, Saudi Arabia

Reviewed by:

Jorge Luis Castillo,

Universidad Peruana Cayetano

Heredia, Peru

Anand Marya,

University of Puthisastra, Cambodia

${ }^{*}$ Correspondence:

Mohamed G. Hassan

mgamal@dent.svu.edu.eg

Specialty section: This article was submitted to

Pediatric Dentistry,

a section of the journal

Frontiers in Dental Medicine

Received: 19 December 2020

Accepted: 24 February 2021

Published: 18 March 2021

Citation:

Yacout YM, Hassan MG,

El-Harouni NM, Ismail HA and Zaher AR (2021) Tooth-Bone-Borne Vs. Bone-Borne Palatal Expanders: A

Systematic Review.

Front. Dent. Med. 2:644002. doi: 10.3389/fdmed.2021.644002

\section{Tooth-Bone-Borne Vs. Bone-Borne Palatal Expanders: A Systematic Review}

\author{
Yomna M. Yacout ${ }^{1}$, Mohamed G. Hassan ${ }^{2,3 *}$, Nadia M. El-Harouni ${ }^{1}$, Hanan A. Ismail ${ }^{1}$ and \\ Abbas R. Zaher ${ }^{1}$ \\ ${ }^{1}$ Department of Orthodontics, Faculty of Dentistry, Alexandria University, Alexandria, Egypt, ${ }^{2}$ Department of Orthodontics, \\ Faculty of Oral and Dental Medicine, South Valley University, Qena, Egypt, ${ }^{3}$ Department of Orthodontics, Faculty of Dentistry, \\ October 6 University, Giza, Egypt
}

The aim of this review was to evaluate the current evidence regarding post-treatment effects of tooth-bone-borne vs. bone-borne expanders. A search was conducted in MEDLINE via PubMed, Web of Science, Scopus, Cochrane Library, Google Scholar, and Open Gray; in addition to a hand search in reference lists of selected articles and creating a search alert in electronic databases. Selection criteria included randomized and prospective clinical trials comparing post-expansion skeletal and/or dento-alveolar effects of tooth-bone-borne expanders to those of bone-borne expanders. Following study retrieval and selection, relevant data was extracted, and risk of bias was assessed using the revised RoB 2 tool for randomized clinical trials. After examining 10 full text articles, one randomized clinical trial was finally included. The study compared the dento-alveolar effects of tooth-bone-borne and bone-borne expanders, following expansion and after 6 months, using digital dental casts. Using the RoB 2 tool, the study was judged overall to show some concerns. A definitive conclusion could not be drawn from this systematic review due to the scarcity of clinical trials tackling the research question. A need for future well-conducted research was highlighted in this review.

Keywords: maxillary expander, maxillary expansion, tooth-bone-borne expander, bone-borne expander, miniscrew-supported expander, skeletal anchorage device

\section{INTRODUCTION}

Transverse maxillary deficiency is a sign of malocclusion that is frequently seen by orthodontists, and is commonly presented in the form of unilateral or bilateral posterior crossbite (1). The prevalence of posterior crossbites was found to range between 8 and $22 \%$ in different populations and different age groups (2-4). It may also be manifested in the form of dental crowding of maxillary arch and presence of wide buccal corridors on smiling $(1,5)$. If transverse maxillary deficiency is left untreated in growing subjects it may lead to other consequences such as mandibular functional shift and asymmetry, altered muscular function, temporomandibular disorders, altered tongue posture, and narrowing of the nasal airway $(1,6)$.

Patients usually undergo palatal expansion as a part of their orthodontic treatment to correct the transverse discrepancy by opening the mid-palatal suture (7). Palatal expansion was first introduced by Angell in 1860 (8), however, it was not until the 1960s that the technique was adopted by American orthodontists when Haas (9-11) reintroduced it. In his design, the expansion screw was embedded in an acrylic pad that was connected to banded molars and premolars (9). 
Since then, different designs of tooth-borne and tooth-tissueborne palatal expansion appliances were proposed in the literature such as hyrax (12), bonded Hyrax (13), modified bonded Hyrax (14), and two-point Hyrax supported on first molars only (15).

Tooth-borne and tooth-tissue-borne appliances transmit their expansion forces to the mid-palatal suture through supporting teeth hence may result in unfavorable effects such as dentoalveolar tipping, root resorption, and periodontal damage in the form of gingival recession or fenestration of buccal bone of supporting teeth (16-18). The dento-alveolar tipping that ensues may result in opening of the bite due to clockwise rotation of the mandible (19). Moreover, the skeletal expansion may not be fully maintained during the retention period following the active expansion (20). In order to overcome such adverse effects, and also to evade surgically-assisted palatal expansion in older patients, miniscrew-supported palatal expanders were introduced $(21,22)$.

There is currently no consensus in the literature regarding the optimal design of miniscrew-supported palatal expanders, but they mainly fall into two categories; either tooth-bone-borne where the expander is supported using miniscrews in addition to anchor teeth, or bone-borne where the expander is only supported using miniscrews without banding teeth (23). The manner and extent of stress distribution, hence the skeletal and dento-alveolar effects of expansion may vary by incorporating anchor teeth in the expander design (24).

Thus, this systematic review was conducted to evaluate the current evidence regarding post-expansion effects of tooth-boneborne expanders in contrast to bone-borne expanders. The null hypothesis was that there is no difference in the treatment outcome between the two types of expanders.

\section{MATERIALS AND METHODS}

\section{Protocol and Registration}

The protocol for this systematic review was submitted to Prospective International Registry of Systematic Reviews (PROSPERO) on April 12 ${ }^{\text {th }}, 2020$ (ID: CRD42020179159). It was updated on July $9^{\text {th }}, 2020$ to broaden the included population to encompass human participants of any age, and to expand the time frame in which outcomes were evaluated. All versions of the protocol are available at https://www.crd.york.ac.uk/prospero. The review was conducted and reported based on Preferred Reporting Items for Systematic Reviews and Meta-Analyses (PRISMA) statement (25).

\section{Eligibility Criteria}

This systematic review was performed in order to answer the following question: "Do changes in maxillary expander design influence the treatment outcomes?"

Based on PICOS (25) approach, the following criteria were applied:

Abbreviations: CBCT, cone-beam computed tomography; PICOS, PopulationIntervention-Comparison-Outcome-Study design; PRISMA, Preferred Reporting Items for Systematic Reviews and Meta-Analyses; PROSPERO, Prospective International Registry of Systematic Reviews; RCT, randomized clinical trial.
- Population (P): human participants of any age or sex, having posterior crossbite or transverse maxillary deficiency.

- Intervention (I): tooth-bone-borne (hybrid) palatal expanders.

- Comparison (C): bone-borne palatal expanders.

- Outcome (O): post-expansion skeletal and/or dento-alveolar effects.

- Study design (S): Randomized clinical trials (RCTs) or non-RCTs.

Literature reviews, systematic reviews, meta-analyses, studies involving cleft lip and palate, medically compromised or syndromic patients, studies combining miniscrew-supported palatal expansion with other treatment (e.g., facemask), or with surgical procedures (e.g., surgically-assisted rapid palatal expansion), animal studies, in vitro studies, and finite element analysis studies were excluded.

\section{Information Sources and Search Strategy}

Studies were identified by searching the electronic databases: MEDLINE via PubMed, Web of Science, Scopus, and the Cochrane library, from the inception of each database up to May 2020. No language or publication date restrictions were applied during the search. In addition, Google scholar and Open Gray were searched for gray literature. Reference lists of relevant articles were manually searched and "Citation Networks" of relevant articles in Web of Science were checked to identify studies that could have been missed in the electronic database searches. A search alert was created for each database using its respective search strategy in order to be notified with any new relevant studies, and alerts were monitored on regular basis until July 2020. The search strategy was first developed for PubMed, then it was adapted to syntax rules of each database as shown in Table 1.

\section{Study Selection}

All potentially relevant titles and abstracts were imported into a reference manager (EndNote X7, Thomson Reuters) and duplicate records were omitted. Titles and abstracts were screened independently by two reviewers ( $\mathrm{MH}, \mathrm{YY})$, and were ranked based on information provided by the title and abstract as "Excluded" or "Potentially eligible." Full text was retrieved for any article graded by at least one of the reviewers as "Potentially eligible." Eligibility assessment was performed on retrieved fulltext articles by the same two reviewers and any disagreement between them was resolved by discussion and consensus. If no agreement could be reached, a third reviewer was consulted (AZ).

\section{Data Collection}

A data extraction form was designed to extract the following information from included studies: author, publication year, study design, study setting, details of participants (sample size, mean age, sex distribution), details of expanders' designs, expansion protocol, assessment method, observation period, and outcomes. One reviewer extracted the data (YY) and another reviewer $(\mathrm{MH})$ revised them. 
TABLE 1 | Literature search conducted to identify studies (last search date May $7^{\text {th }}, 2020$ ).

\begin{tabular}{|c|c|c|}
\hline Database & Search & Search strategy \\
\hline \multirow[t]{3}{*}{ MEDLINE (via PubMed) } & \#1 & "palatal expansion technique"[MeSH Terms] OR maxillary expan*[Title/Abstract] OR palatal expan[Title/Abstract] \\
\hline & \#2 & $\begin{array}{l}\text { "orthodontic anchorage procedures"[MeSH Terms] OR miniscrew-supported[Title/Abstract] OR } \\
\text { miniscrew-assisted[Title/Abstract] OR miniscrew*[Title/Abstract] OR mini-screw*[Title/Abstract] OR } \\
\text { mini-implant*[Title/Abstract] OR microimplant*[Title/Abstract] OR micro-implant*[Title/Abstract] OR } \\
\text { bone-borne[Title/Abstract] OR bone-anchor*[Title/Abstract] OR skeletal anchorage[Title/Abstract] OR } \\
\text { skeletally-anchored[Title/Abstract] }\end{array}$ \\
\hline & \#3 & \#1 AND \#2 \\
\hline \multirow[t]{3}{*}{ Scopus } & \#1 & TITLE-ABS-KEY (“maxillary expan*” OR “palatal expan*”) \\
\hline & \#2 & $\begin{array}{l}\text { TITLE-ABS-KEY (“miniscrew-supported" OR "miniscrew-assisted" OR miniscrew OR "mini screw" OR "mini } \\
\text { implant" OR microimplant OR "micro implant" OR "bone borne" OR "bone anchor" OR "skeletal" anchor*") }\end{array}$ \\
\hline & \#3 & \#1 AND \#2 \\
\hline \multirow[t]{3}{*}{ Cochrane } & \#1 & [mh "palatal expansion techniques"] OR maxillary NEXT expan*:ti,ab,kw OR palatal NEXT expan*:ti,ab,kw \\
\hline & \#2 & $\begin{array}{l}\text { [mh "orthodontic anchorage procedures"] OR miniscrew-supported:ti,ab,kw OR miniscrew-assisted:ti,ab,kw OR } \\
\text { miniscrew:ti,ab,kw OR miniscrews:ti,ab,kw OR mini-screw:ti,ab,kw OR mini-screws:ti,ab,kw OR } \\
\text { mini-implant:ti,ab,kw OR mini-implants:ti,ab,kw OR microimplant:ti,ab,kw OR microimplants:ti,ab,kw OR } \\
\text { micro-implant:ti,ab,kw OR micro-implants:ti,ab,kw OR bone-borne:ti,ab,kw OR bone-anchored:ti,ab,kw OR } \\
\text { skeletal anchorage:ti,ab,kw OR skeletally-anchored:ti,ab,kw }\end{array}$ \\
\hline & \#3 & \#1 AND \#2 \\
\hline \multirow[t]{3}{*}{ Web of Science } & $\# 1$ & maxillary-expan* OR palatal-expan* \\
\hline & \#2 & $\begin{array}{l}\text { miniscrew-supported OR miniscrew-assisted OR miniscrew OR mini-screw OR mini-implant* OR microimplant* } \\
\text { OR micro-implant* OR bone-borne OR bone-anchor* OR skeletal anchorage OR skeletally-anchored }\end{array}$ \\
\hline & \#3 & \#1 AND \#2 \\
\hline Google scholar & & $\begin{array}{l}\text { allintitle: "maxillary OR palatal expander OR expansion" AND (miniscrew OR mini-screw OR mini-implant OR } \\
\text { microimplant OR micro-implant OR bone-borne OR bone-anchored OR bone-anchorage OR skeletal-anchorage } \\
\text { OR skeletally-anchored) }\end{array}$ \\
\hline \multicolumn{3}{|c|}{ https://scholar.google.com.eg/ } \\
\hline OpenGrey & & $\begin{array}{l}\text { ("maxillary expander" OR "maxillary expansion" OR "palatal expander" OR "palatal expansion") AND ("miniscrew } \\
\text { supported" OR "miniscrew assisted" OR miniscrew OR miniscrews OR "mini screw" OR "mini screws" OR "mini } \\
\text { implant" OR "mini implants" OR microimplant OR microimplants OR "micro implant" OR "micro implants" OR } \\
\text { "bone borne" OR "bone anchored" OR "bone anchorage" OR "skeletal anchorage" OR "skeletally anchored") }\end{array}$ \\
\hline
\end{tabular}
miniscrew-assisted[Title/Abstract] OR miniscrew*[Title/Abstract] OR mini-screw*[Title/Abstract] OR mimplant ${ }^{\star}[$ Title/Abstract] OR microimplant*[Title/Abstract] OR micro-implant*[Title/Abstract] OR skeletally-anchored[Title/Abstract]

359

3404

5010

283

337

\footnotetext{
*,\#These values are part of the search strategies that were applied in the named databases. The bold values represent the final number of records that match the search in each database.
}

\section{Risk of Bias in Individual Studies}

Risk of bias assessment was done independently by two reviewers (MH, YY) using Cochrane Collaboration's revised RoB 2 tool (26) for RCTs. Bias arising from randomization process; bias due to deviations from intended interventions; bias due to missing data; bias in outcome measurement; and bias in selection of reported results were assessed. If information was missing in any domain, authors of the trial were contacted for clarification. An overall risk of bias of the study was set as "High" if any of the domains was judged as "High risk;" it was set as "Low" if all domains were judged as "low risk," and it was set as "Some concerns" if at least one domain was judged as "Some concerns." Any disagreements between the two reviewers were solved through arbitration by a third reviewer $(\mathrm{AZ})$.

\section{RESULTS}

\section{Study Selection}

The process of study identification and screening is shown in Figure 1. Electronic database search resulted in identification of 957 records, in addition to 95 other records identified through other sources. After removing 538 duplicates, a total of 514 records were screened by title and abstract to identify potentially eligible articles. Screening resulted in exclusion of 504 records, and full texts of the remaining 10 articles were retrieved and analyzed according to the eligibility criteria. Nine articles were excluded for the following reasons: One study was a letter to the editor (27), two studies were retrospective $(28,29)$, three studies were reviews (30-32), and three studies examined only one type of miniscrew-supported palatal expander (33-35). Consequently, one study (36) was deemed eligible for inclusion in the systematic review.

\section{Study Characteristics}

Characteristics of the included study are summarized in Table 2. The selected study was an RCT that involved 53 adolescent patients having 5-10 $\mathrm{mm}$ transverse maxillary deficiency, with 47 patients included in final analysis. Two designs of miniscrewsupported palatal expanders were compared to each other and to a conventional expander. The hybrid expander was supported using 2 miniscrews placed anteriorly between first and second premolars, in addition to the banded first molars, whereas the 


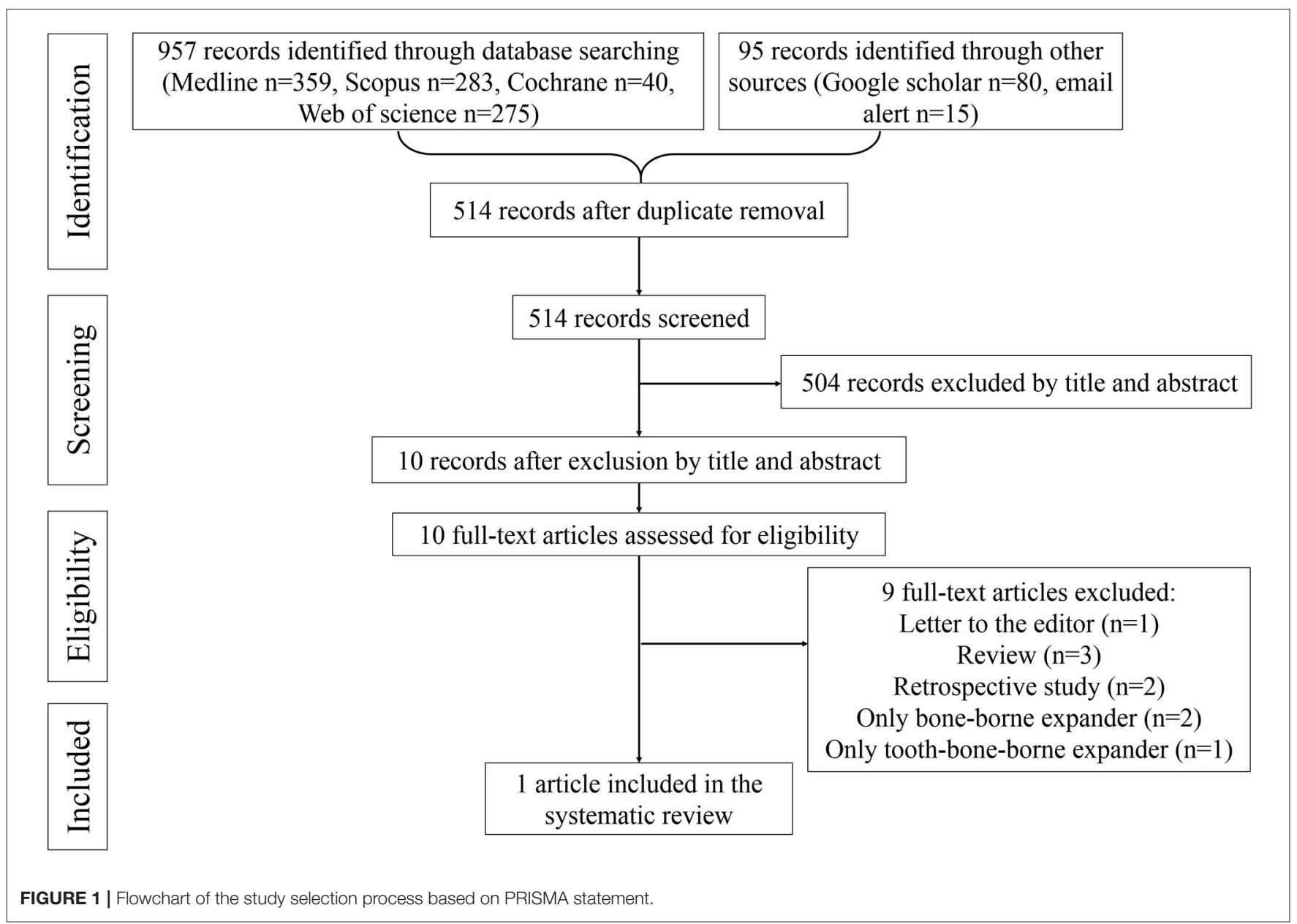

bone-borne expander was supported using only 4 miniscrews. The same activation protocol was used for the 3 expanders. The outcome of the study was post-expansion and post-retention dento-alveolar effects assessed using digital dental casts. Changes in the maxillary arch were assessed by superimposition of the casts then recording positional changes in the coordinates of the teeth, and logarithmically calculating the teeth angulations. On the other hand, linear interdental measurements were conducted on mandibular casts.

\section{Risk of Bias}

The risk of bias for the included study is summarized in Figure 2. Using RoB 2 tool, the study was judged overall to show some concerns. This judgment was reached because although the authors mentioned that sample randomization was undertaken, they did not specify the method of random sequence generation or allocation concealment. Moreover, the study was not prospectively registered, and no protocol was provided to allow evaluation of bias in selection of the reported result.

\section{Results of Individual Studies}

No information was provided in the study regarding the initial amount of transverse maxillary deficiency in each group, and the endpoint of expansion was not defined. The mean amount of expansion was 7.07 and $6.22 \mathrm{~mm}$ in the hybrid group and boneborne group, respectively, and it was not found to be different between the groups. Both appliances showed a statistically significant buccal movement of maxillary molars post-expansion. The mean buccal movement of right first molar mesio-buccal cusp was $2.46( \pm 1.61)$ and $0.74( \pm 0.82) \mathrm{mm}$ in the hybrid and bone-borne groups, respectively, while that of the left first molar was $2.32( \pm 1.54)$ and $1.92( \pm 1.17) \mathrm{mm}$ in the hybrid and bone-borne groups, respectively. The authors reported significantly more buccal movement of the right first molar following expansion with hybrid appliance compared to boneborne appliance, which persisted post-retention. Both appliances showed a statistically significant buccal movement of maxillary first premolars post-expansion, but it was not significantly different between the two groups. The mean post-expansion buccal movement of right first premolar buccal cusp was 1.22 $( \pm 1.20)$ and $0.48( \pm 0.58) \mathrm{mm}$ in the hybrid and bone-borne groups, respectively, whereas that of the left first premolar was $1.51( \pm 1.95)$ and $1.97( \pm 0.90) \mathrm{mm}$ in the hybrid and bone-borne groups, respectively. The maxillary right $\left(6.50^{\circ} \pm 4.65\right)$ and left $\left(4.31^{\circ} \pm 3.86\right)$ first molars and right first premolar $\left(3.08^{\circ} \pm 4.87\right)$ showed significant buccal tipping following expansion using 
TABLE 2 | Characteristics of the included study.

\begin{tabular}{|c|c|c|c|c|c|c|}
\hline $\begin{array}{l}\text { Author; } \\
\text { publication year }\end{array}$ & $\begin{array}{l}\text { Study design; } \\
\text { study setting; } \\
\text { country }\end{array}$ & $\begin{array}{l}\text { Sample size }(F / M) ; \\
\text { mean age }\end{array}$ & $\begin{array}{l}\text { Expander design and } \\
\text { expansion protocol }\end{array}$ & $\begin{array}{l}\text { Assessment method; } \\
\text { observation period }\end{array}$ & $\begin{array}{l}\text { Amount of } \\
\text { expansion }^{\S} \text {; } \\
\text { expansion } \\
\text { duration }^{\S \S \S}\end{array}$ & Outcome measure \\
\hline $\begin{array}{l}\text { Canan and } \\
\text { Senişik; } 2017 \text { (36) }\end{array}$ & $\begin{array}{l}\text { RCT; Süleyman } \\
\text { Demirel University; } \\
\text { Turkey }\end{array}$ & $\begin{array}{l}\mathrm{TBG}=16(8 / 8) ; 12.63 \pm \\
1.36 \\
\mathrm{BBG}=16(9 / 7) ; 12.92 \pm \\
1.07 \\
\mathrm{HG}=15(8 / 7) ; 13.41 \pm \\
0.88\end{array}$ & $\begin{array}{l}\text { TBG: bands on first premolars } \\
\text { and first molars BBG: } 4 \\
\text { miniscrews placed on the } \\
\text { palatal slope bilaterally } \\
\text { between first and second } \\
\text { premolars, and between } \\
\text { second premolars and first } \\
\text { molars HG: } 2 \text { miniscrews } \\
\text { placed on the palatal slope } \\
\text { bilaterally between first and } \\
\text { second premolars, and } 2 \\
\text { bands on first molars } \\
\text { All appliances activated twice } \\
\text { per day ( } 0.25 \text { mm per turn) }\end{array}$ & $\begin{array}{l}\text { Digital dental casts; } \\
\text { Pre-treatment, } \\
\text { Post-treatment, } 6 \\
\text { months Post-retention } \\
\end{array}$ & $\begin{array}{l}\mathrm{TBG}=6.59 \pm 1.28 \\
13.31 \pm 2.78 \\
\mathrm{BBG}=6.22 \pm 0.66 \\
12.44 \pm 1.31 \\
\mathrm{HG}=7.07 \pm 1.25 \\
14.13 \pm 2.50\end{array}$ & $\begin{array}{l}\text { Linear and angular } \\
\text { dental movements }\end{array}$ \\
\hline
\end{tabular}

BBG, Bone-borne group; F, Female; HG, Hybrid group; M, Male; RCT, Randomized clinical trial; TBG, Tooth-borne group.

$\S$ Age in years.

$\S \S$ Amount of expansion in $\mathrm{mm}$.

$\S \S \S$ Expansion duration in days.

\begin{tabular}{|l|l|l|l|l|l|}
\hline D1 & D2 & D3 & D4 & D5 & Overall \\
\hline$?$ & + & + & + & $?$ & $?$ \\
\hline
\end{tabular}

\section{Domains}

\section{D1: Bias arising from the randomization process \\ D2: Bias due to deviations from intended interventions \\ D3: Bias due to missing outcome data}

D4: Bias in measurement of the outcome

\section{D5: Bias in selection of the reported result}

FIGURE 2 | Summary of the risk of bias assessment according to Cochrane Collaboration's RoB 2 tool.

hybrid appliance, which was significantly uprighted during retention period. In contrast, no significant buccal tipping was evident post-expansion using the bone-borne appliance. The mandibular inter-molar width was found to significantly increase in both bone-borne and hybrid groups following expansion, but no difference was found between the groups.

Regarding observed complications, more loosened appliances were reported in the bone-borne group (25\%) compared to the hybrid group (7\%). Conversely, more broken appliances were reported in the hybrid group (13\%) compared to the bone-borne group (6\%). Two patients experienced loosened miniscrews in the bone-borne group. On the other hand, no failures were reported in the hybrid group.

\section{DISCUSSION}

\section{Summary of Evidence}

The aim of this systematic review was to evaluate evidence from randomized and prospective clinical trials on postexpansion effects of tooth-bone-borne and bone-borne palatal 
expanders. Recent systematic reviews have examined the use of miniscrew-supported palatal expansion, but none of them compared the effects of combining dental and skeletal anchorage as opposed to only using skeletal anchorage in the expander design (23, 37-39). This systematic review is, to our knowledge, the first to compare the effects of tooth-bone-borne vs. boneborne expanders. The principal findings were that both designs resulted in significant expansion of maxillary molars and premolars, with significant molar tipping taking place with the tooth-bone-borne expander.

Although hybrid and bone-borne expanders were initially described more than a decade ago $(22,40)$, remarkably limited research has been carried out to compare both designs, and often the results were not in accord. The search through literature yielded three studies that compared the two designs; two were retrospective studies that used cone-beam computed tomography (CBCT) for comparison $(28,29)$, and one was an RCT that employed digital dental casts (36). Ultimately, only the RCT by Canan and Senişik (36) was included in the current systematic review. Since only one study was found to conform to the eligibility criteria, only a narrative synthesis was performed. The included study, referred to by its authors as "Part 1," only compared dento-alveolar effects of the expansion appliances (36). A search for a second part of the study did not yield any results, and the authors were contacted by email for clarification but no response was obtained.

Significant expansion of maxillary molars was observed by Canan and Senişik (36) in both groups, but a significant difference between the two groups was only found on the right side. Increased maxillary inter-molar width was previously reported with various designs of miniscrew-supported maxillary expanders using CBCT (40-43). Moon et al. (28) reported in their retrospective CBCT study a significantly greater dental expansion in hybrid expanders compared to bone-borne expanders, but no significant difference in skeletal expansion between the two groups. Significant buccal movement of premolars was observed in both groups despite not being attached to any of the appliances (36). This can be explained by the movement of premolars with their skeletal base during expansion. Increased inter-premolar width agrees with previous research using CBCT $(41,43)$.

Although Canan and Senişik (36) did not find statistically significant inter-group differences regarding dental tipping, significant post-expansion buccal tipping of maxillary molars was reported with hybrid expanders but not with bone-borne expanders. This result is considerably expected because molars are included in the design of hybrid expanders, contrary to the bone-borne expanders. A similar result immediately after expansion was reported by $\mathrm{Oh}$ et al. (29) in their retrospective CBCT study, where slightly less buccal tipping of molars was observed with bone-borne expander compared to hybrid expander. Similarly, Moon et al. (28) found significant increase in molar buccal tipping in the hybrid group, but not the bone-borne group, 3 months post-expansion. The right first premolar showed significant buccal tipping following expansion using hybrid expander despite not being directly attached to the appliance (36). A possible reason could be alveolar bone bending or rotation of the maxillary halves that take place during expansion (44). Subsequent post-retention uprighting of molars and premolars was reported in the study, and was explained by the authors by pressure from the cheeks and forces of occlusion (36).

More clinical complications were reported with bone-borne expanders compared to hybrid expanders (36). Loss of stability of the appliances or their supporting miniscrews possibly took place in the bone-borne group because all the expansion force fell on the miniscrews, while in the hybrid group the anchor teeth might have impeded the instability (24). If the miniscrews fail, an advantage of the hybrid design is that the attachment between the molars and the expander may act as a safety mechanism against swallowing or aspiration (45). Another asset of the hybrid expander is that the appliance may act as a surgical guide thus facilitating the insertion of miniscrews (46).

\section{Limitations}

Evidence from the included RCT should be interpreted with caution because it showed some concerns regarding risk of bias. There were some concerns arising from the randomization process and regarding the selection of the reported results. An attempt was made to contact the authors for clarification regarding these concerns, however, no response was received.

The reviewed article only compared dento-alveolar effects of miniscrew-supported expanders, and no consideration was given to skeletal effects. In addition, despite reporting the mean amount of expansion in each group, no information was provided regarding baseline severity of transverse maxillary deficiency, or the endpoint of expansion. Another drawback of this review is that only adolescents were assessed in the included study. Age and skeletal maturity may affect the amount and pattern of maxillary expansion, as well as relapse (41). Hence, results may differ if the study is conducted on older patients.

\section{Recommendations for Future Research}

Future research should compare skeletal as well as dento-alveolar effects of miniscrew-supported maxillary expanders in different age groups and using reliable assessment methods such as CBCT and digital casts. Other variables that should be considered include number of incorporated anchor teeth, number and size of miniscrews, and their site of placement. The patients' perception of the different designs is another domain that should be considered. The study critiqued in this systematic review had some methodological shortcomings. Therefore, researchers should focus on high-quality RCTs, with sufficient power and robust methodology.

\section{CONCLUSION}

A definitive conclusion cannot be drawn from this systematic review due to the scarcity of clinical trials tackling the research question. However, based on the available evidence, both tooth-bone-borne and bone-borne expanders produce significant maxillary dento-alveolar expansion, with less complications encountered in the tooth-bone-borne expander. There is a need for further well-conducted research comparing both appliance designs. 


\section{DATA AVAILABILITY STATEMENT}

The raw data supporting the conclusions of this article will be made available by the authors, without undue reservation.

\section{AUTHOR CONTRIBUTIONS}

YY and MH conceived and designed the review, were the major contributors in writing the manuscript, and performed study selection. YY extracted data and MH revised them.

\section{REFERENCES}

1. McNamara JA. Maxillary transverse deficiency. Am J Orthod Dentofacial Orthop. (2000) 117:567-70. doi: 10.1016/S0889-5406(00)70202-2

2. Alhammadi MS, Halboub E, Fayed MS, Labib A, El-Saaidi C. Global distribution of malocclusion traits: a systematic review. Dental Press J Orthod. (2018) 23:40.e1-10. doi: 10.1590/2177-6709.23.6.40.e1-10.onl

3. Tausche E, Luck O, Harzer W. Prevalence of malocclusions in the early mixed dentition and orthodontic treatment need. Eur J Orthod. (2004) 26:23744. doi: $10.1093 /$ ejo/26.3.237

4. Petrén S, Bondemark L, Söderfeldt B. A systematic review concerning early orthodontic treatment of unilateral posterior crossbite. Angle Orthod. (2003) 73:588-96. doi: 10.1043/0003-3219(2003)073<0588:ASRCEO >2.0.CO;2

5. Maulik C, Nanda R. Dynamic smile analysis in young adults. Am J Orthod Dentofacial Orthop. (2007) 132:307-15. doi: 10.1016/j.ajodo.2005.11.037

6. Madian A, El-Harouni N, Abdallah E. Maxillary symmetry, mandibular symmetry and condylar position in functional unilateral posterior cross bite patients. Egypt Orthodon J. (2015) 48:11-22. doi: 10.21608/eos.2015.78699

7. Korbmacher H, Huck L, Merkle T, Kahl-Nieke B. Clinical profile of rapid maxillary expansion-outcome of a national inquiry. J Orofac Orthop. (2005) 66:455-68. doi: 10.1007/s00056-005-0440-5

8. Angell E. Treatment of irregularities of the permanent or adult teeth. Dent Cosmos. (1860) 1:599-600.

9. Haas AJ. Rapid expansion of the maxillary dental arch and nasal cavity by opening the midpalatal suture. Angle Orthod. (1961) 31:73-90.

10. Haas AJ. The treatment of maxillary deficiency by opening the midpalatal suture. Angle Orthod. (1965) 35:200-17.

11. Haas AJ. Palatal expansion: just the beginning of dentofacial orthopedics. Am J Orthod. (1970) 57:219-55. doi: 10.1016/0002-9416(70)90241-1

12. Biederman W. A hygienic appliance for rapid expansion. J Pract Orthod. (1968) 2:67-70.

13. Cohen M, Silverman E. A new and simple palate splitting device. J Clin Orthod. (1973) 7:368-9.

14. Howe RP. Palatal expansion using a bonded appliance. Report of a case. Am J Orthod. (1982) 82:464-8. doi: 10.1016/0002-9416(82)90313-X

15. Schneidman E, Wilson S, Erkis R. Two-point rapid palatal expansion: an alternate approach to traditional treatment. Pediatr Dent. (1990) 12:92-7.

16. Lo Giudice A, Galletti C, Gay-Escoda C, Leonardi R. CBCT assessment of radicular volume loss after rapid maxillary expansion: a systematic review. $J$ Clin Exp Dent. (2018) 10:e484-e94. doi: 10.4317/jced.54745

17. Kartalian A, Gohl E, Adamian M, Enciso R. Cone-beam computerized tomography evaluation of the maxillary dentoskeletal complex after rapid palatal expansion. Am J Orthod Dentofacial Orthop. (2010) 138:48692. doi: 10.1016/j.ajodo.2008.10.025

18. Lo Giudice A, Barbato E, Cosentino L, Ferraro CM, Leonardi R. Alveolar bone changes after rapid maxillary expansion with tooth-born appliances: a systematic review. Eur J Orthod. (2018) 40:296-303. doi: 10.1093/ejo/cjx057

19. Wertz RA. Skeletal and dental changes accompanying rapid midpalatal suture opening. Am J Orthod. (1970) 58:41-66. doi: 10.1016/0002-9416(70)90127-2

20. Schiffman PH, Tuncay OC. Maxillary expansion: a meta analysis. Clin Orthod Res. (2001) 4:86-96. doi: 10.1034/j.1600-0544.2001.040205.x

21. Lee KJ, Park YC, Park JY, Hwang WS. Miniscrew-assisted nonsurgical palatal expansion before orthognathic surgery for a patient with severe
AZ was the third reviewer in case of disagreements. $\mathrm{NE}-\mathrm{H}, \mathrm{HI}$, and AZ edited and revised the manuscript. All authors contributed to the article and approved the submitted version.

\section{ACKNOWLEDGMENTS}

YY wishes to thank Dr. Hassan Kassem (Lecturer of Orthodontics, Faculty of Dentistry, Alexandria University, Egypt) for his insightful comments and suggestions. mandibular prognathism. Am J Orthod Dentofacial Orthop. (2010) 137:8309. doi: 10.1016/j.ajodo.2007.10.065

22. Wilmes B, Nienkemper M, Drescher D. Application and effectiveness of a mini-implant- and tooth-borne rapid palatal expansion device: the hybrid hyrax. World J Orthod. (2010) 11:323-30.

23. Krüsi M, Eliades T, Papageorgiou SN. Are there benefits from using bone-borne maxillary expansion instead of tooth-borne maxillary expansion? A systematic review with meta-analysis. Prog Orthod. (2019) 20:9. doi: 10.1186/s40510-019-0261-5

24. Seong EH, Choi SH, Kim HJ, Yu HS, Park YC, Lee KJ. Evaluation of the effects of miniscrew incorporation in palatal expanders for young adults using finite element analysis. Korean J Orthod. (2018) 48:819. doi: 10.4041/kjod.2018.48.2.81

25. Moher D, Liberati A, Tetzlaff J, Altman DG. Preferred reporting items for systematic reviews and meta-analyses: the PRISMA statement. PLoS Med. (2009) 6:e1000097. doi: 10.1371/journal.pmed.1000097

26. Sterne JAC, Savović J, Page MJ, Elbers RG, Blencowe NS, Boutron I, et al. RoB 2: a revised tool for assessing risk of bias in randomised trials. BMJ. (2019) 366:14898. doi: 10.1136/bmj.14898

27. Winsauer H, Walter A, Scherfler M, Ploder O. What are the limits of microimplant-assisted palatal expanders? Am J Orthod Dentofacial Orthop. (2017) 151:3-4. doi: 10.1016/j.ajodo.2016.09.011

28. Moon HW, Kim MJ, Ahn HW, Kim SJ, Kim SH, Chung KR, et al. Molar inclination and surrounding alveolar bone change relative to the design of bone-borne maxillary expanders: a СВCT study. Angle Orthod. (2020) 90:13-22. doi: 10.2319/050619-316.1

29. Oh H, Park J, Lagravere-Vich MO. Comparison of traditional RPE with two types of micro-implant assisted RPE: CBCT study. Semin Orthod. (2019) 25:60-8. doi: 10.1053/j.sodo.2019.02.007

30. Suzuki H, Moon W, Previdente LH, Suzuki SS, Garcez AS, Consolaro A. Miniscrew-assisted rapid palatal expander (MARPE): the quest for pure orthopedic movement. Dental Press J Orthod. (2016) 21:17-23. doi: 10.1590/2177-6709.21.4.017-023.oin

31. Lee K-J, Choi S-H, Choi T-H, Shi K-K, Keum B-T. Maxillary transverse expansion in adults: rationale, appliance design, and treatment outcomes. Semin Orthod. (2018) 24:52-65. doi: 10.1053/j.sodo.2018. 01.006

32. Gurgel J. MARPE - quais os efeitos esperados? Rev Clín Ortod Dental Press. (2019) 18:60-6. doi: 10.14436/1676-6849.18.2.060-066.epa

33. Vassar JW, Karydis A, Trojan T, Fisher J. Dentoskeletal effects of a temporary skeletal anchorage device-supported rapid maxillary expansion appliance (TSADRME): a pilot study. Angle Orthod. (2016) 86:2419. doi: 10.2319/013015-76.1

34. Farhangfar A, Bogowicz P, Heo G, Lagravère MO. Palatal bone resorption in bone-anchored maxillary expander treatment. Int Orthod. (2012) 10:27488. doi: $10.1016 /$ j.ortho. 2012.06 .005

35. Lagravère MO, Gamble J, Major PW, Heo G. Transverse dental changes after tooth-borne and bone-borne maxillary expansion. Int Orthod. (2013) 11:21-34. doi: 10.1016/j.ortho.2012.12.003

36. Canan S, Senişik NE. Comparison of the treatment effects of different rapid maxillary expansion devices on the maxilla and the mandible. Part 1: evaluation of dentoalveolar changes. Am J Orthod Dentofacial Orthop. (2017) 151:1125-38. doi: 10.1016/j.ajodo.2016.11.022 
37. Algharbi M, Bazargani F, Dimberg L. Do different maxillary expansion appliances influence the outcomes of the treatment? Eur J Orthod. (2018) 40:97-106. doi: 10.1093/ejo/cjx035

38. Khosravi M, Ugolini A, Miresmaeili A, Mirzaei H, ShahidiZandi V, Soheilifar S, et al. Tooth-borne versus bone-borne rapid maxillary expansion for transverse maxillary deficiency: a systematic review. Int Orthod. (2019) 17:425-36. doi: 10.1016/j.ortho.2019. 06.003

39. Copello FM, Marañón-Vásquez GA, Brunetto DP, Caldas LD, Masterson D, Maia LC, et al. Is the buccal alveolar bone less affected by mini-implant assisted rapid palatal expansion than by conventional rapid palatal expansion? - A systematic review and meta-analysis. Orthod Craniofac Res. (2020) 23:23749. doi: 10.1111/ocr.12374

40. Lagravère MO, Carey J, Heo G, Toogood RW, Major PW. Transverse, vertical, and anteroposterior changes from bone-anchored maxillary expansion vs traditional rapid maxillary expansion: a randomized clinical trial. Am J Orthod Dentofacial Orthop. (2010) 137:304.e1-12; discussion 3045. doi: 10.1016/j.ajodo.2009.09.016

41. Akin M, Akgul YE, Ileri Z, Basciftci FA. Three-dimensional evaluation of hybrid expander appliances: a pilot study. Angle Orthod. (2016) 86:816. doi: 10.2319/121214-902.1

42. Celenk-Koca T, Erdinc AE, Hazar S, Harris L, English JD, Akyalcin S. Evaluation of miniscrew-supported rapid maxillary expansion in adolescents: a prospective randomized clinical trial. Angle Orthod. (2018) 88:7029. doi: 10.2319/011518-42.1

43. Gunyuz Toklu M, Germec-Cakan D, Tozlu M. Periodontal, dentoalveolar, and skeletal effects of tooth-borne and tooth-bone-borne expansion appliances. Am J Orthod Dentofacial Orthop. 148:97-109. doi: 10.1016/j.ajodo.2015.02.022

44. Cantarella D, Dominguez-Mompell R, Moschik C, Mallya SM, Pan HC, Alkahtani MR, et al. Midfacial changes in the coronal plane induced by microimplant-supported skeletal expander, studied with cone-beam computed tomography images. Am J Orthod Dentofacial Orthop. (2018) 154:337-45. doi: 10.1016/j.ajodo.2017.11.033

45. Mosleh MI, Kaddah MA, Abd ElSayed FA, ElSayed HS. Comparison of transverse changes during maxillary expansion with 4-point bone-borne and tooth-borne maxillary expanders. Am J Orthod Dentofacial Orthop. (2015) 148:599-607. doi: 10.1016/j.ajodo.2015.04.040

46. Cantarella D, Savio G, Grigolato L, Zanata P, Berveglieri C, Giudice AL, et al. A new methodology for the digital planning of micro-implantsupported maxillary skeletal expansion. Med Devices (Auckl). (2020) 13:93106. doi: 10.2147/MDER.S247751

Conflict of Interest: The authors declare that the research was conducted in the absence of any commercial or financial relationships that could be construed as a potential conflict of interest.

Copyright (C) 2021 Yacout, Hassan, El-Harouni, Ismail and Zaher. This is an openaccess article distributed under the terms of the Creative Commons Attribution License (CC BY). The use, distribution or reproduction in other forums is permitted, provided the original author(s) and the copyright owner(s) are credited and that the original publication in this journal is cited, in accordance with accepted academic practice. No use, distribution or reproduction is permitted which does not comply with these terms. 